\title{
Starspots and relativity: Applied Doppler imaging for the Gravity Probe B mission ${ }^{\star}$
}

\author{
S.C. Marsden ${ }^{1,2, \star \star}$, S.V. Berdyugina ${ }^{2}$, J.-F. Donati ${ }^{3}$, J.A. Eaton ${ }^{4}$, and M.H. Williamson ${ }^{4}$ \\ 1 Anglo-Australian Observatory, PO Box 296, Epping, NSW 1710, Australia \\ 2 Institute of Astronomy, ETH Zurich, CH-8092, Switzerland \\ 3 Laboratoire d'Astrophysique, Observatoire Midi-Pyrénées, F-31400 Toulouse, France \\ ${ }^{4}$ Center of Excellence in Information Systems, Tennessee State University, Nashville TN 37209, USA
}

Received 2007 Sep 3, accepted 2007 Oct 27

Published online 2007 Dec 15

\begin{abstract}
Key words stars: imaging - stars: individual (IM Pegasi) - stars: rotation - starspots
We present Doppler images and surface differential rotation measurements for the primary of the RS CVn binary IM Pegasi, the guide star for the Gravity Probe B experiment. The data used is a subset of that taken during optical support of the mission and was obtained almost nightly over a near three year period from the Automatic Spectroscopic Telescope operated by Tennessee State University. Using the technique of least-squares deconvolution to increase the signal-to-noise ratio of the data, we have reconstructed 31 maximum entropy Doppler images of the star. The images show that the spot features are relatively stable for over a year (and possibly longer) with both a polar spot and lower latitude features. The most intense features are located on the side facing the secondary. In addition, we have incorporated a solar-like differential rotation law into the imaging process to determine the level of surface differential rotation for IM Peg for 22 epochs. A weighted least-squares average of the measurements gives a surface shear of $0.0142 \pm 0.0007 \mathrm{rad} / \mathrm{d}$, meaning that the equator takes $\sim 440 \pm 20$ days to lap the poles. Although the level of surface differential rotation was shown to vary over the period of the observations, this may indicate an underestimate in the errors of the method rather than any temporal evolution in the differential rotation.
\end{abstract}

(c) 2007 WILEY-VCH Verlag GmbH \& Co. KGaA, Weinheim

\section{Introduction}

IM Pegasi (HR 8703, HD 216489) is a RS CVn binary with an orbital period of approximately 25 days. The most recent determination of the orbital parameters of the system is given by Marsden et al. (2005) which gives the system a circular orbit. The primary component of the system was determined by Berdyugina, Ilyin \& Tuominen (1999) to be a K2 giant star with Marsden et al. (2005) giving the first detection of the secondary. The stellar parameters of the primary and secondary of IM Peg are given in Table 1.

Gravity Probe B (GP-B) is a polar orbiting satellite ${ }^{1}$ designed to test two predictions of Einstein's theory of general relativity. The geodetic effect due to the curvature of space near the Earth, and the Lense-Thirring or "frame-dragging" effect due to the rotation of the Earth. The motions are measured with respect to the optical centroid of the satellite's guide star, IM Pegasi. IM Peg was chosen as it is optically bright in both radio and optical wavelengths so that its motion can be tracked relative to nearby guide quasars and the satellite's small guide telescope can accurately track on the star. Given the accuracy required of the experiment all pos-

\footnotetext{
$\star$ Movies are available via http://www.aip.de/AN/movies

$\star \star$ Corresponding author: scm@aao.gov.au

1 Further information on the Gravity Probe B mission can be found at http://einstein.stanford.edu/. It was launched in 2004 April, with the science phase covering from 2004 September to 2005 September.
}

sible contributions to the motion of the optical centroid of IM Peg must be measured or bounded. In support of the GP-B mission we undertook extensive optical observations of IM Peg to determine what extent surface spot activity on the primary could affect the optical centroid of the star.

Table 1 Stellar parameters for the primary and secondary of the IM Peg system. Those values marked with ${ }^{\mathrm{a}}$ are from Berdyugina et al. (1999), those with ${ }^{\mathrm{b}}$ are from Marsden et al. (2005), and those with ${ }^{\mathrm{c}}$ are from Lebach et al. (1999). The other parameters have been determined from this work.

\begin{tabular}{lc}
\hline Parameter & Value $(\mathrm{P}+\mathrm{S})$ \\
\hline Spectral Type & $\mathrm{K}^{\mathrm{IIII}} \mathrm{a}^{\mathrm{a}}+$ early GV \\
Mass & $1.8 \pm 0.2 \mathrm{M}_{\odot}^{\mathrm{b}}+1.0 \pm 0.1 \mathrm{M}_{\odot}^{\mathrm{b}}$ \\
Radius & $13.3 \pm 0.6 \mathrm{R}_{\odot}^{\mathrm{a}}+1.00 \pm 0.07 \mathrm{R}_{\odot}^{\mathrm{b}}$ \\
Luminosity & $54 \pm 9 \mathrm{~L}_{\odot}^{\mathrm{b}}+0.9 \pm 0.3 \mathrm{~L}_{\odot}^{\mathrm{b}}$ \\
$T_{\text {eff }}$ & $4450 \pm 50 \mathrm{~K}^{\mathrm{a}}+5650 \pm 200 \mathrm{~K}^{\mathrm{b}}$ \\
Orbital period & $24.64877 \pm 0.00003 \mathrm{~d}^{\mathrm{b}}$ \\
Orbital radius $\left(a_{1} \sin i\right)$ & $16.19 \pm 0.06 \mathrm{R}_{\odot}^{\mathrm{c}}$ \\
Distance & $96 \pm 3 \mathrm{pc}^{\mathrm{c}}$ \\
Inclination angle & $70 \pm 10^{\circ}$ \\
$v \sin i$ & $27.0 \pm 0.5 \mathrm{~km} \mathrm{~s}^{-1}+\lesssim 3 \mathrm{~km} \mathrm{~s}^{-1}$ \\
\hline
\end{tabular}

\section{Observations and data reduction}

Optical observations of IM Peg in support of GP-B have been undertaken on a number of telescopes. However, 
this paper deals with results presented at the $5^{\text {th }}$ Potsdam thinkshop held at the Astrophysikalisches Institut Potsdam 2007 June 24 to 29 . These results have used only data obtained from one of the telescopes, the Automatic Spectroscopic Telescope (AST, Eaton \& Williamson 2004) operated by Tennessee State University. This data set is the largest of those collected for IM Peg and spans almost 3 years. A forthcoming paper will present results from the complete data set.

IM Peg was observed nightly (when possible) using the AST at Fairborn Observatory in Arizona. The AST is a 2-m Cassegrain telescope under computer control, equipped with a white-pupil fiber-fed èchelle spectrograph. The observations gave complete wavelength coverage from 4920 to $7110 \AA$ and cover from 2003 August to 2005 May. In mid-September 2004 a new fiber was installed on the system, markedly increasing the system throughput.

As the $\mathrm{S} / \mathrm{N}$ of the individual spectra was too low for single-lined Doppler imaging (average 100) we used Least-Squares Deconvolution (LSD, Donati et al. 1997) to combine $\sim 2700$ photospheric lines in each èchelle spectrum into a single high-S/N profile. The LSD line mask was created from the Kurucz atomic database and ATLAS9 atmospheric models (Kurucz 1993) for a photospheric temperature of $4500 \mathrm{~K}$ and a surface gravity of $\log g=2.5$, very similar to the IM Peg primary values found by Berdyugina et al. (1999). The average S/N of the LSD profiles is $\sim 700$.

By fitting a Gaussian to the secondary's LSD profile we were able to remove the secondary's contribution to the primary's LSD profile.

\section{Results}

\subsection{Image reconstruction}

The LSD profiles of IM Peg were phased to the ephemeris of Marsden et al. (2005) and 31 surface images of IM Peg were created. An example of one of the images is shown in Fig. 1. An animated GIF of the complete sequence of AST images showing the spot evolution of IM Peg over the complete observational period is given in Movie 1 and can be found on the AN website ${ }^{2}$. The cycle numbers represent the number of rotational cycles of the star since $\mathrm{T}_{\text {conj }}$ assuming that the orbital and rotational periods are equal.

The images were reconstructed using the Doppler imaging code of Brown et al. (1991) and Donati \& Brown (1997), which implements the Skilling \& Bryan (1984) algorithm for maximum entropy reconstruction. This technique produces images with the minimum amount information (surface features) required to reproduce the observations. The code uses a two component temperature model, one for the spots and one for the photosphere.

As the number of observations for each rotational cycle is usually not complete enough to create an accurate

\footnotetext{
2 http://www.aip.de/AN/movies
}

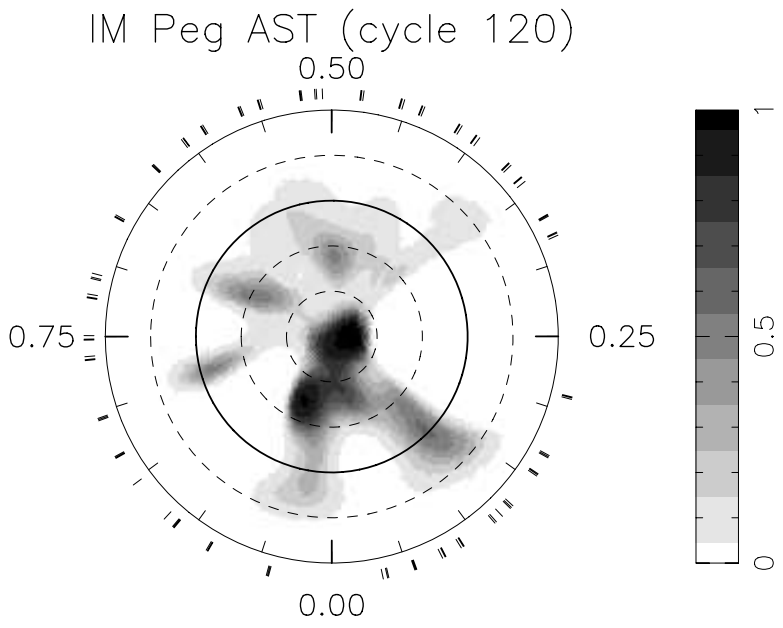

Fig. 1 Maximum entropy brightness image reconstruction of IM Peg for rotational cycle 120 from the AST data. The image is a flattened polar projection extending down to a latitude of $-60^{\circ}$. The bold line denotes the equator and the radial ticks around the image indicate the phases at which the star was observed.

Doppler image, each image has been recreated from data covering two stellar rotations. For example the image in Fig. 1 (rotational cycle 120) has been created from data spanning phases 119.5 to 121.5 , thus the midpoint of cycle 120 actually corresponds to phase 120.5 . This also means that the images are created from overlapping data sets.

\subsection{Surface differential rotation}

In order to determine the surface differential rotation of IM Peg a simplified solar-like differential rotation law has been incorporated into the imaging process:

$$
\Omega(l)=\Omega_{\mathrm{eq}}-d \Omega \sin ^{2} l \quad[\mathrm{rad} / \mathrm{d}],
$$

where $\Omega(l)$ is the rotation rate at latitude $l, \Omega_{\mathrm{eq}}$ is the equatorial rotation rate, and $d \Omega$ is the rotational shear between the equator and the poles.

By treating $\Omega_{\mathrm{eq}}$ and $d \Omega$ as free parameters an image is created for each pair of values and the fit to the data is determined by $\chi^{2}$-minimisation. Fitting a paraboloid to the resultant $\chi^{2}$-landscape, the best fitting values (including $1 \sigma$ errors) for $\Omega_{\mathrm{eq}}$ and $d \Omega$ are determined. This process is outlined further in Petit, Donati \& Collier Cameron (2002).

A differential rotation measurement was attempted for each pair of images we had available and resulted in 22 measurements of surface differential rotation for IM Peg. The results are summarized in Fig. 2.

\section{Discussion and conclusions}

The images of IM Peg shown in Movie 1 span 2.7 years, corresponding to 40 rotations of the star (with some rotations missing due to the periodic unobservability of IM Peg). The images show that IM Peg has a polar spot and lower latitude features at almost all latitudes down to $-30^{\circ}$. Other 


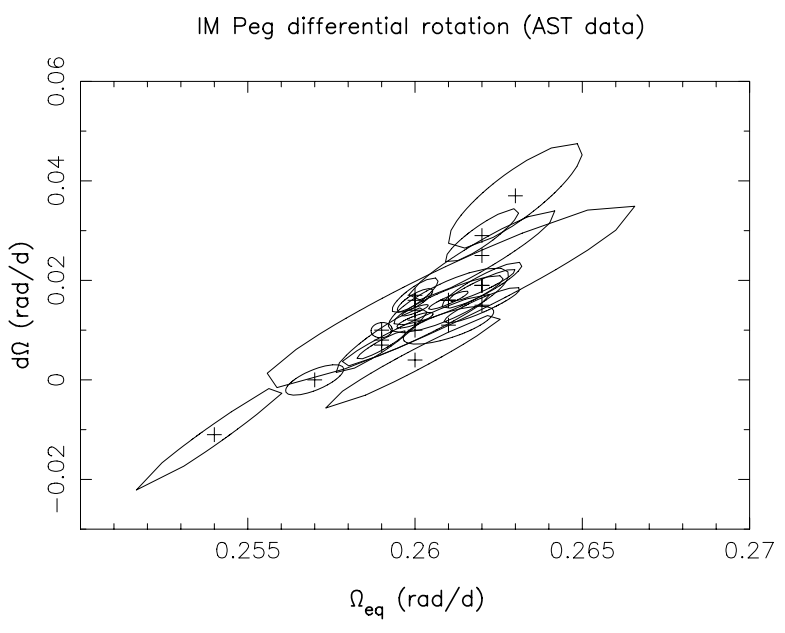

Fig. 2 Surface differential rotation parameters for the 22 measurements obtained for IM Peg. The points are marked with a plus sign with the ellipses showing the $1 \sigma$ confidence intervals.

than the polar feature the main spot group appears to be located around phase 0.0 , which corresponds to the side facing the secondary. Given that the radius of the IM Peg primary is a significant fraction of the orbital radius (Table 1), this could be due to some interaction of the secondary with the primary's magnetic field.

Due to the poor throughput of the fiber on the AST prior to mid-September 2004, the quality of the reconstructed images is poorer for those images earlier than cycle 118 . Therefore, just looking at the images from cycles 118 to 138 (covering 21 rotations and 1.4 years) shows the spot features on IM Peg to be reasonably stable during this time. The main evolution is an observed rotation of the features. This indicates that the equatorial rotation period and orbital period of the system are not equal.

The scatter in the differential rotation measurements of IM Peg shown in Fig. 2 is significantly greater than the $1 \sigma$ errors of most measurements. Some of this scatter may well be due other evolution in the spot features, such as new spots appearing (although, as mentioned, the images are relatively stable) and for a couple of measurements the limited number of observations may also have an effect. However, the scatter in the differential rotation measurements is still significant and does not appear to follow any trend with time. Could this mean that the error bars in the method we have used are underestimated? Further tests, including making sure that the datasets used to determine differential rotation are completely independent (as suggested by Andrew Collier Cameron), will have to be done before this is certain.

All but one of the 22 differential rotation measurements show a normal solar-type differential rotation (i.e. a positive value of $d \Omega$ ) with the equator rotating more rapidly than the poles. Thus it would appear that IM Peg does not have antisolar differential rotation.

A weighted least-squares average (where the weight of each data point is $1 / \sigma^{2}$ ) of the differential rotation measurements (ignoring the 3 most outlying values in $\Omega_{\mathrm{eq}}$ and $d \Omega$ )

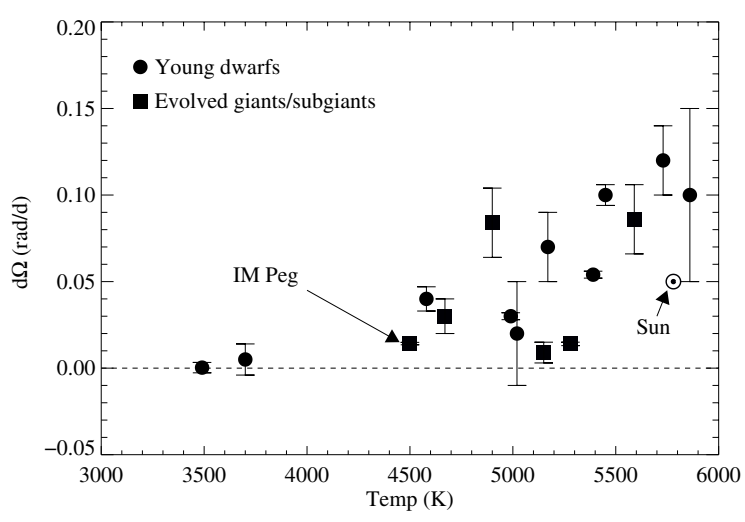

Fig. 3 Surface differential rotation vs. stellar temperature for both active young dwarfs and evolved (sub)giant stars. Based on data from Barnes et al. (2005) and Pascal Petit (priv. comm.).

gives $\Omega_{\text {eq }}=0.2606 \pm 0.0002 \mathrm{rad} / \mathrm{d}$ and $d \Omega=0.0142 \pm$ $0.0007 \mathrm{rad} / \mathrm{d}$. This equates to an equatorial rotation period of $24.11 \pm 0.02$ days and means the equator laps the poles every $\sim 440 \pm 20$ days.

It was shown by Barnes et al. (2005) that $d \Omega$ increases with stellar temperature (and hence mass) for young dwarf stars, but what about more evolved stars such as IM Peg? Figure 3 plots the data from Barnes et al. (2005) and includes some more recent measurements of (sub)giant stars provided by Pascal Petit (private communication). Where more than one measurement of a star exists a weighted least-squares average has been used. Although the increase is clearly seen for young dwarfs, the results for evolved (sub)giant stars are less clear. This may be due to the smaller number of measurements, but may also be due to the fact that all these (sub)giant stars are in binary systems, where effects such as tidal locking may well have an impact.

Acknowledgements. This work was partly funded by NASA through Stanford University and the Smithsonian Astrophysical Observatory as well as ETH Research Grant TH-3/05-1.

\section{References}

Barnes, J.R., Collier Cameron, A., Donati, J.-F., James, D.J., Marsden, S.C., Petit, P.: 2005, MNRAS 357, L1

Berdyugina, S.V., Ilyin, I., Tuominen, I.: 1999, A\&A 347, 932

Brown, S.F., Donati, J.-F., Rees, D.E., Semel, M.: 1991, A\&A 250, 463

Donati, J.-F., Brown, S.F.: 1997, A\&A 326, 1135

Donati, J.-F., Semel, M., Carter, B.D., Rees, D.E., Cameron, A.C.: 1997, MNRAS 291, 658

Eaton, J.A., Williamson, M.H.: 2004, Proc. SPIE 5496, 710

Kurucz, R.: 1993, CDROMs 13 and 18, SAO, Cambridge

Lebach, D.E., Ratner, M.I., Shapiro, I.I., Ranson, R.R., Bietenholz, M.F., Lestrade, J.-F.: 1999, ApJ 517, L43

Marsden, S.C., Berdyugina, S.V., Donati, J.-F., et al.: 2005, ApJ 634, L173

Petit, P., Donati, J.-F., Collier Cameron, A.: 2002. MNRAS 334, 374

Skilling, J., Bryan, R.K.: 1984, MNRAS 211, 111 